\title{
Direct Visualization and Manipulation of Stacking Orders in Few-Layer Graphene by Dynamic Atomic Force Microscopy
}

Hongjian $\mathrm{Wu}^{\dagger}$, Xiaoxiang $\mathrm{Yu}^{\dagger}$, Mengjian Zhu ${ }^{*}$, , Zhihong Zhu ${ }^{\ddagger}$, Jianyu Zhang ${ }^{\S}$, Sen Zhang $^{\dagger}$, Shiqiao Qin ${ }^{*}+$, Guang Wang ${ }^{\dagger}$, Gang Peng ${ }^{\dagger}$, Jiayu Dai ${ }^{*}$, , Kostya S. Novoselov

${ }^{\dagger}$ Department of Physics, National University of Defense Technology, Changsha 410073, Hunan, China.

$\$$ College of Advanced Interdisciplinary Studies \& Hunan Provincial Key Laboratory of Novel Nano-Optoelectronic Information Materials and Devices, National University of Defense Technology, Changsha 410073, Hunan, China.

$\S$ Institute of Electronic Engineering, China Academy of Engineering Physics, Mianyang 621900, Sichuan, China

" Department of Materials Science and Engineering, National University of

Singapore, Singapore 117575, Singapore

${ }^{\perp}$ Chongqing 2D Materials Institute, Liangjiang New Area, Chongqing 400714, China

*(M.Z.) Email zhumengjian11@ nudt.edu.cn. 
*(S.Q.) Email sqqin8@ @udt.edu.cn.

*(J.D.) Email jydai@ nudt.edu.cn.

\section{Section 1. Sample preparation and characterization.}

Section 2. Molecular dynamics simulation.

Figure S1. Raman spectra of typical FLG samples.

Figure S2. AFM morphology images and corresponding height profiles of typical FLG samples.

Figure S3. Phase shift and difference of ABA- and ABC-stacked FLG.

Figure S4. Characterizations of a 12-layer FLG sample.

Figure S5. Tapping mode AFM phase image, topography image and height image of FLG.

Figure S6. Calculated potential energy between the AFM tip and ABA- or ABCstacked TLG.

Figure S7. The atomic radial distribution function (RDF) between the tip and TLG.

Figure S8. Raman images of FLG before and after the tapping of AFM tip.

Figure S9. Simulation system for the phase transition.

Figure S10. Stress analysis in FLG under the tapping of the AFM tip.

Figure S11. Average temperature of upper layer graphene under the tapping of the AFM tip.

Figure S12. MD simulation snapshots of system with larger sample width and inplane tip-DW distance 
Table S1. Phase shift and difference of ABA- and ABC-stacked FLG.

Table S2. Comparison between calculation and experiment of phase shift in FLG.

Table S3. The comparison of settings of two simulations.

Movie S1. Enlarged perspective view of atomic structure during one tapping process.

Movie S2. Enlarged perspective view of $\sigma_{\mathrm{zz}}$ stress profile during one tapping process.

Movie S3. Front view of ABC-to-ABA structural phase transition process.

Movie S4. Top view of ABC-to-ABA structural phase transition process.

\section{Section 1. Sample preparation and characterization}

The FLG samples were mechanically exfoliated from the bulk graphite onto a silicon wafer covered with a $285 \mathrm{~nm}$-thick silicon oxide layer. The number of layers of FLG was determined by combining optical microscopy, Raman spectroscopy and atomic force microscopy. Raman spectroscopy of FLG samples were obtained by a commercial confocal Raman spectrometer (Witec Alpha 300R). The laser wavelength is $532 \mathrm{~nm}$ with a laser beam spot size $\sim 0.6 \mu \mathrm{m}$ and the power of the laser through a 50 $\times$ objective lens was fixed at $2 \mathrm{~mW}$ to avoid any thermal heating and laser thinning effect. The Raman mode of the silicon substrate at $520 \mathrm{~cm}^{-1}$ was applied to calibrate the Raman spectroscopy. The grating of Raman spectroscopy was 600-grooves per $\mathrm{mm}$. The morphology and phase images of FLG samples were obtained by a dynamic atomic force microscopy (NT-MDT Solver Pro). The probe is a silicon tip with a radius of curvature of less than $10 \mathrm{~nm}$ and the force constant is $10 \mathrm{~N} / \mathrm{m}$ to $130 \mathrm{~N} / \mathrm{m}$. All images were generated using the tapping mode in the ambient condition at room 
temperature. The topographical and phase image of each sample was obtained simultaneously. All the collected data was analyzed through NOVA SPM software. For the non-invasive AFM phase imaging, as shown in Figure 1, the FLG samples were scanned by a tapping-mode AFM in the ambient condition at room temperature. The topographical and phase image of each sample was obtained simultaneously. All the collected data was analyzed through NOVA SPM software. The setpoints are 11.2 $\mathrm{V}$ for Figure 1g (trilayer), $20 \mathrm{~V}$ for Figure 1h (6-layer), and $10 \mathrm{~V}$ (15-layer); the scan speeds are $1 \mathrm{~Hz}$ per line scanning $(70 \mu \mathrm{m} / \mathrm{s})$ for Figure $1 \mathrm{~g}$ (trilayer), $2 \mathrm{~Hz}(60 \mu \mathrm{m} / \mathrm{s})$ for Figure 1h (6-layer), and $1 \mathrm{~Hz}(80 \mu \mathrm{m} / \mathrm{s})$ for Figure 1i (15-layer), respectively. The corresponding generator frequencies are $336 \mathrm{kHz}, 267 \mathrm{kHz}$, and $311 \mathrm{kHz}$, respectively. In our AFM facility (NT-MDT Solver Pro), it is difficult to determine the exact value of the applied forced at the semi-contact tapping-mode. The applied force is about $1 \mathrm{nN} \sim 13 \mathrm{nN}$ estimated from the setpoint and the force constant of the silicon tip. For the domain wall movement experiments, as shown in Figure 3, the characterization parameters were $12 \mathrm{~V}$ (setpoint), $0.4 \mathrm{~Hz}, 32 \mu \mathrm{m} / \mathrm{s}$ (scan speed), 300 $\mathrm{kHz}$ (generator frequency) before the stacking order transformation and then changed to $10 \mathrm{~V}$ (setpoint), $0.2 \mathrm{~Hz}, 16 \mu \mathrm{m} / \mathrm{s}$ (scan speed), $300 \mathrm{kHz}$ (generator frequency) to trigger the $\mathrm{ABC}$-to-ABA structural phase transition. To drive the propagation of $\mathrm{ABC} / \mathrm{ABA}$ domain walls, we intentionally reduced the setpoint to increase the applied force and increased the scanning period to enhance the tip-sample interaction. The local control of the domain geometry and domain walls movement was achieved by adjusting the trajectory of the AFM tip. 


\section{Section 2. Molecular dynamics simulation}

The molecular dynamics simulation system consists of a silicon AFM tip, a trilayer graphene, and a monolayer graphene as the substrate. The tip is a pillar with rectangular cross-section of $2 \mathrm{~nm} \times 2 \mathrm{~nm}$ and a hemispherical end of $2 \mathrm{~nm}$ diameter. The MD simulation were performed using the LAMMPS package. ${ }^{1}$ We use OVITO package to visualize the atomic structures and trajectories in MD simulations. ${ }^{2}$ The interatomic potentials used for silicon are original Tersoff potential. ${ }^{3}$ The intra-layer, and inter-layer interactions of graphene are described by Tersoff potential, ${ }^{4}$ and registry-dependent interlayer potential. ${ }^{5-6}$ The interactions between silicon and graphene are van der Waals forces modeled by the 12-6 Lennard-Jones potential with parameters $\varepsilon=8.909 \mathrm{meV}$ and $\sigma=3.629 \AA .^{7}$ The cutoffs for Si-C and $\mathrm{C}-\mathrm{C}$ are $16 \AA$. The interlayer distance of graphene is $3.35 \AA$. The lattice constants are $5.442 \AA$ for Si and $2.4919 \AA$ for graphene. The time step is set as $0.1 \mathrm{fs}$, which is short enough to capture the dynamics.

For adhesive energy calculation, the box size is $4.75 \mathrm{~nm} \times 4.98 \mathrm{~nm} \times 8 \mathrm{~nm}$, as shown in Figure S5. The Si tip is moved vertically toward graphene with $0.1 \AA$ per step, and the values of interactional potential energy from distance of $20 \AA$ to $2 \AA$ are calculated meanwhile. For tip-driven phase transition simulation, the box size is 28.48 $\mathrm{nm} \times 4.98 \mathrm{~nm} \times 8 \mathrm{~nm}$, as shown in Figure S7. The taping speed is constant as $1 \AA / \mathrm{fs}$, and the taping amplitude is $18 \AA$, with vertical distance ranging from $2 \AA$ to $20 \AA$. The horizontal distance between tip and domain wall is $50 \AA$. The wrinkle at the middle is the domain wall, the left and right side are ABA-stacked and ABC-stacked 
FLG domains, respectively.
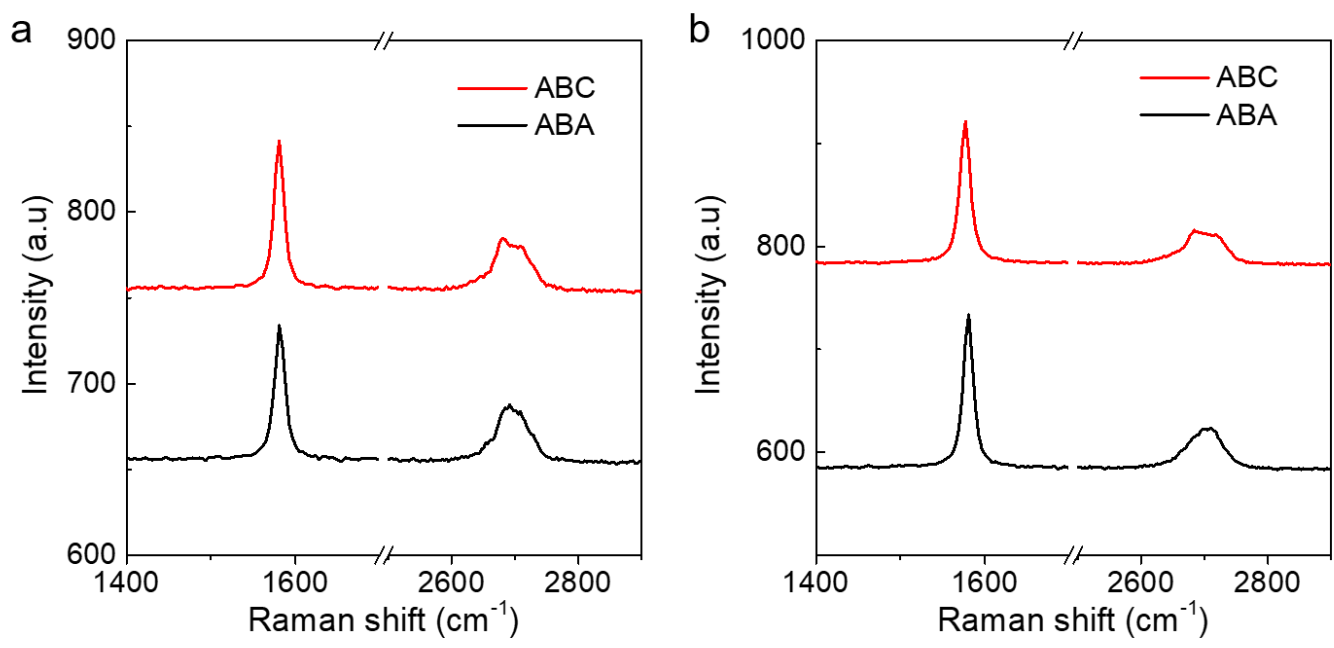

Figure S1. Raman spectra of a 6-layer FLG (sample \#100) (a) and a 15-layer FLG (sample \#36) (b), respectively.
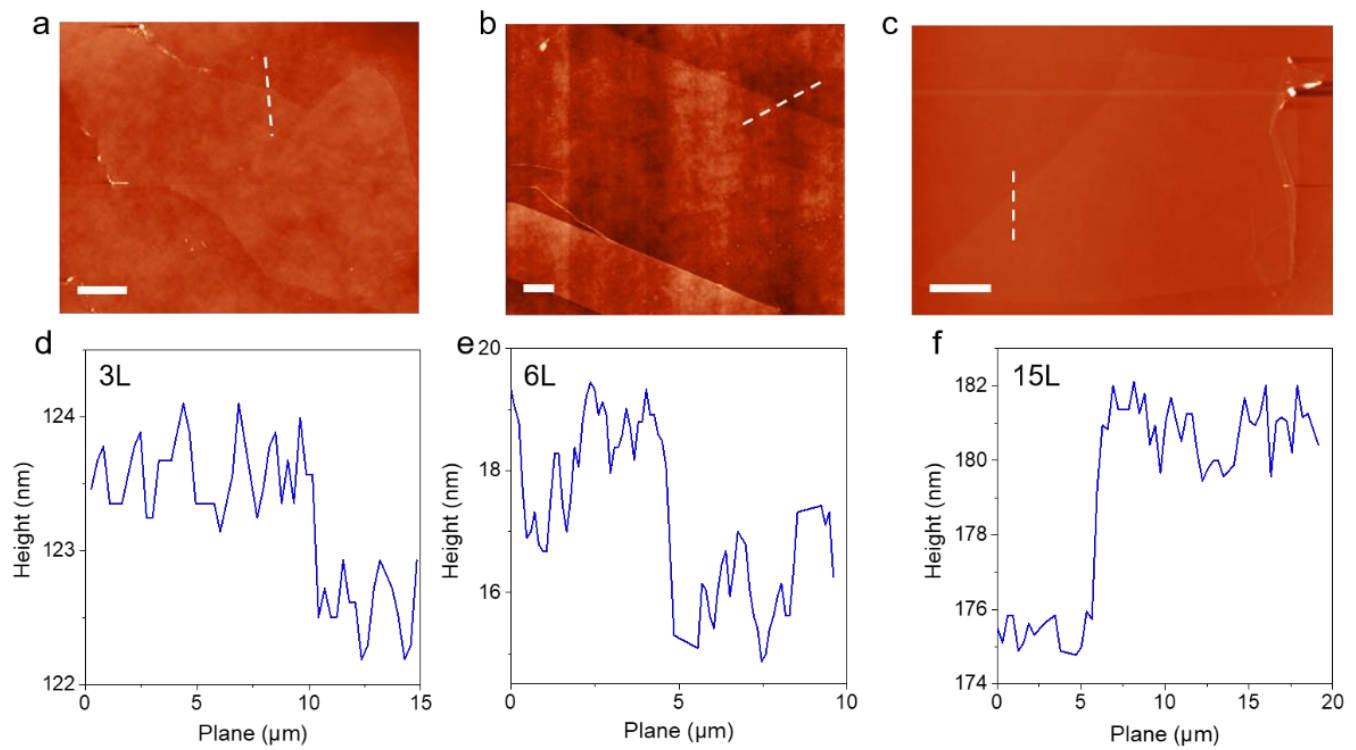

Figure S2. AFM morphology images (a-c) and corresponding height profiles (d-f) of a TLG (sample \#199 (a, d), 6-layer FLG (sample \#100) (b, e) and a 15-layer FLG (sample \#36) (c, f), respectively. The scale bars are $10 \mu \mathrm{m}$. The white dashed lines mark the height profile measurements.

Table S1. Phase shift and difference of ABA- and ABC-stacked FLG 


\begin{tabular}{ccccc}
\hline $\begin{array}{c}\text { Sample } \\
\text { no. }\end{array}$ & $\begin{array}{c}\text { Number } \\
\text { of layers }\end{array}$ & $\begin{array}{c}\text { Phase shift } \\
\text { ABA }\end{array}$ & $\begin{array}{c}\text { Phase shift } \\
\text { ABC }\end{array}$ & $\begin{array}{c}\Delta \varphi \\
\text { (degree) }\end{array}$ \\
\hline 36 & 15 & 11.0 & 4.0 & 7.0 \\
166 & 9 & 81.5 & 79 & 2.5 \\
199 & 3 & 40.9 & 40.7 & 0.2 \\
219 & 6 & 29.8 & 29.5 & 0.3 \\
293 & 9 & 40 & 30 & 10 \\
373 & 3 & 27 & 25.5 & 1.5 \\
393 & 12 & 8.4 & 8 & 0.4 \\
\hline
\end{tabular}
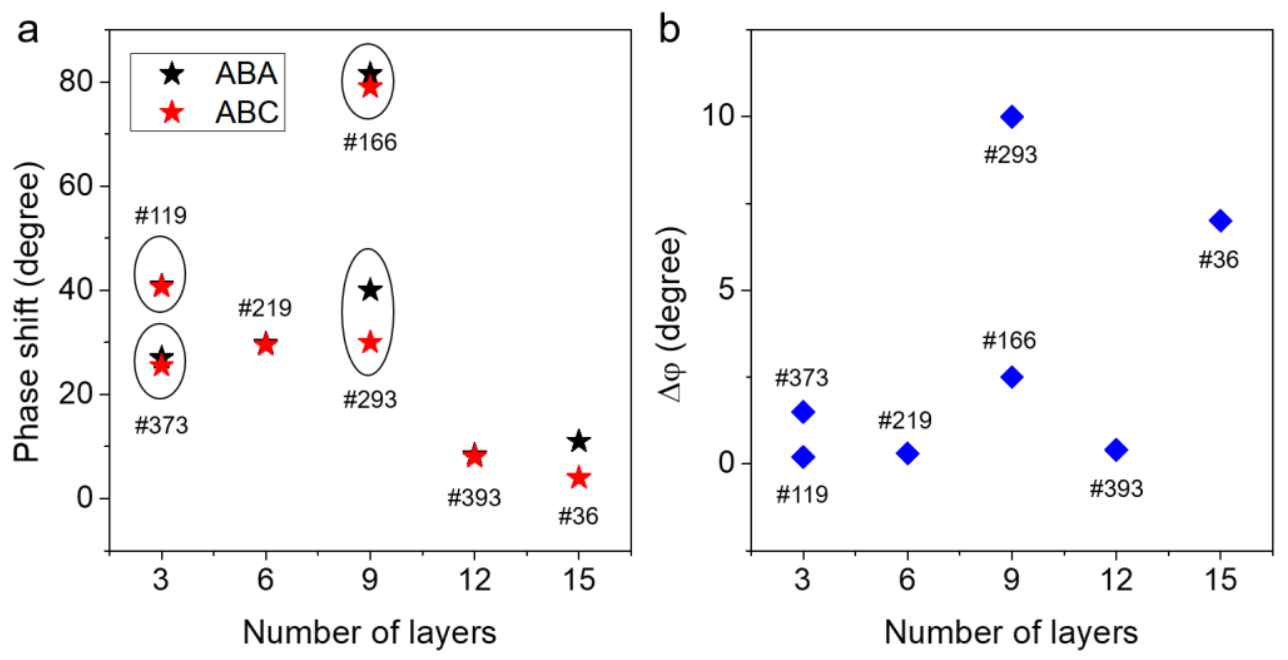

Figure S3. Phase shift (a) and difference (b) of ABA- and ABC-stacked FLG samples with different number of layers from 3 to 15 , respectively. 

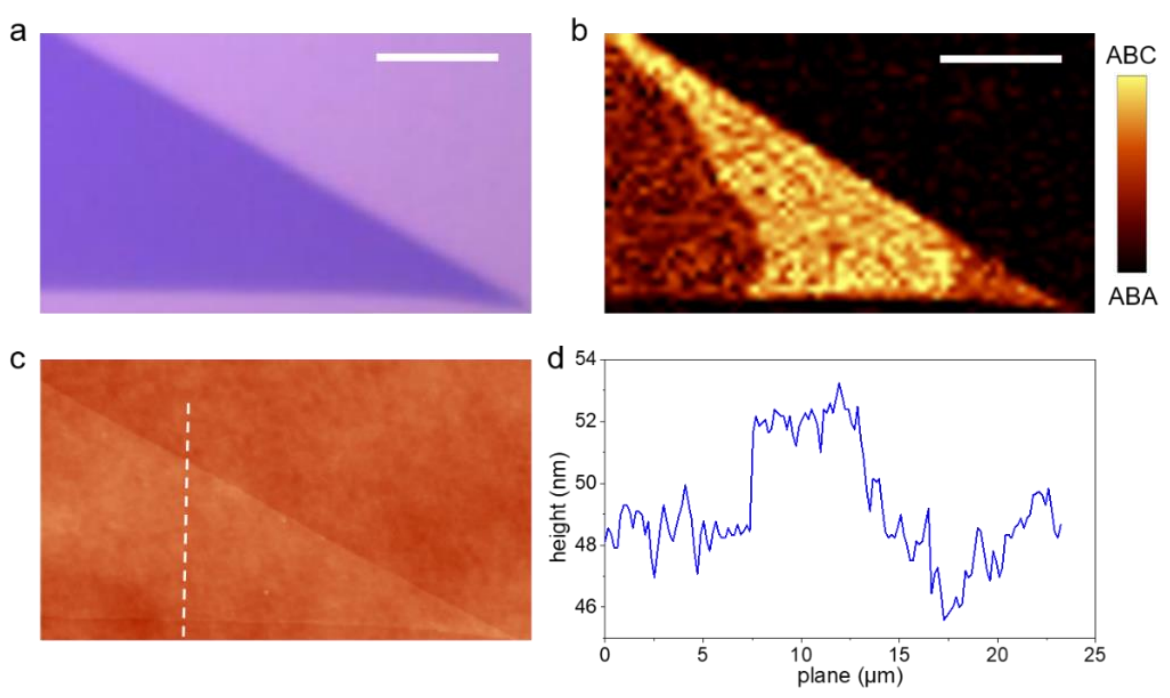

Figure S4. Characterizations of a 12-layer FLG (sample \#393). (a) optical microscopy image, (b) Raman map of integrated intensity of G mode, showing ABAstacked and ABC-stacked domains. The scale bars are $10 \mu \mathrm{m}$. (c) Raman height image and (d) corresponding height profile. The white dashed lines mark the height profile measurements. 

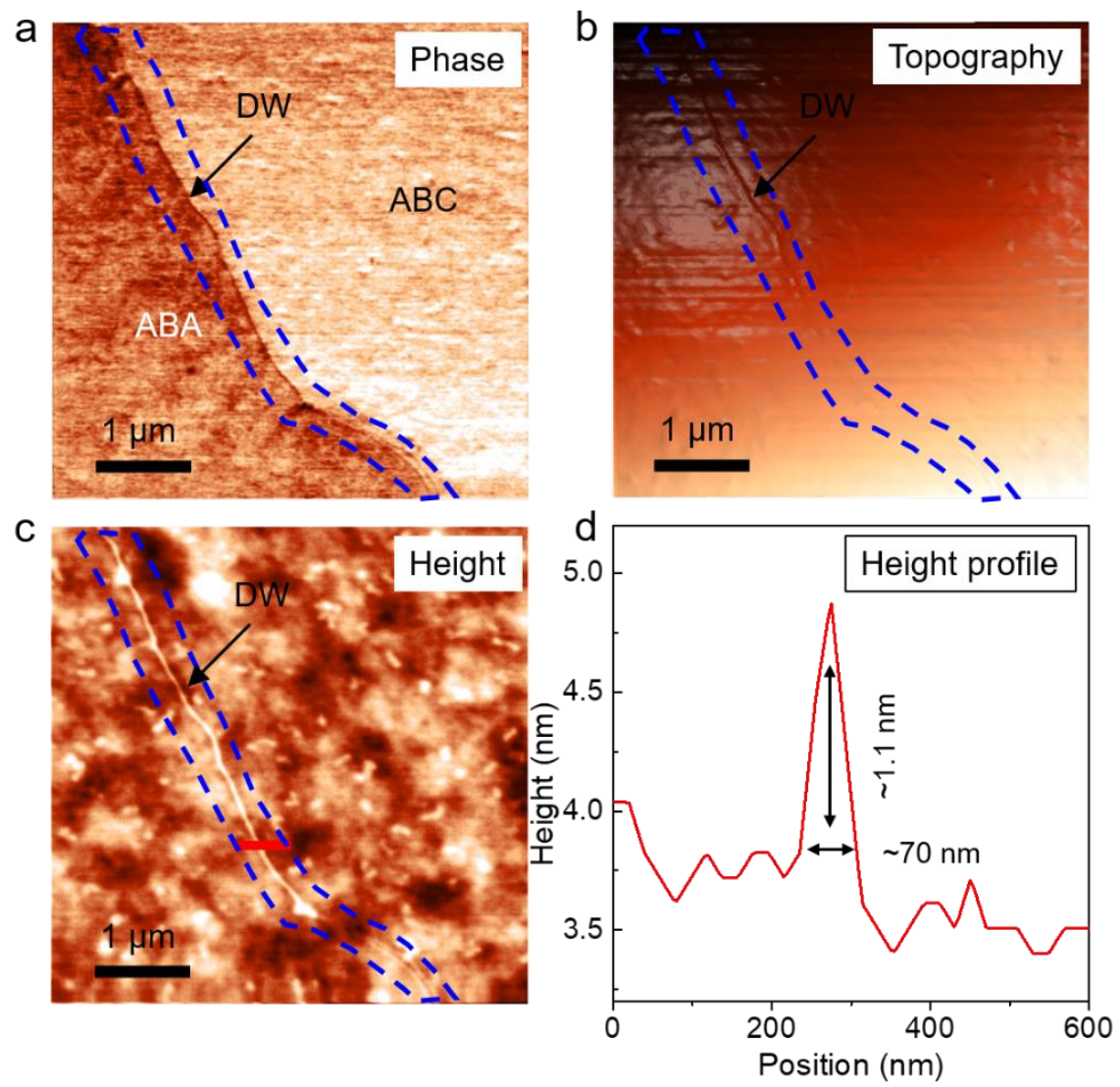

Figure S5. Tapping mode AFM phase image (a), topography image (b) and height image (c) of a 12-layer graphene, which containing ABA- and ABC-stacked domains and the ABA/ABC DW between them. The black arrows and the blue dashed zones highlight the existence of wrinkle-like ABA/ABC DW. (d) The height profile crossing the DW taken from the red line in (c), clearly showing a wrinkle at the DW. 


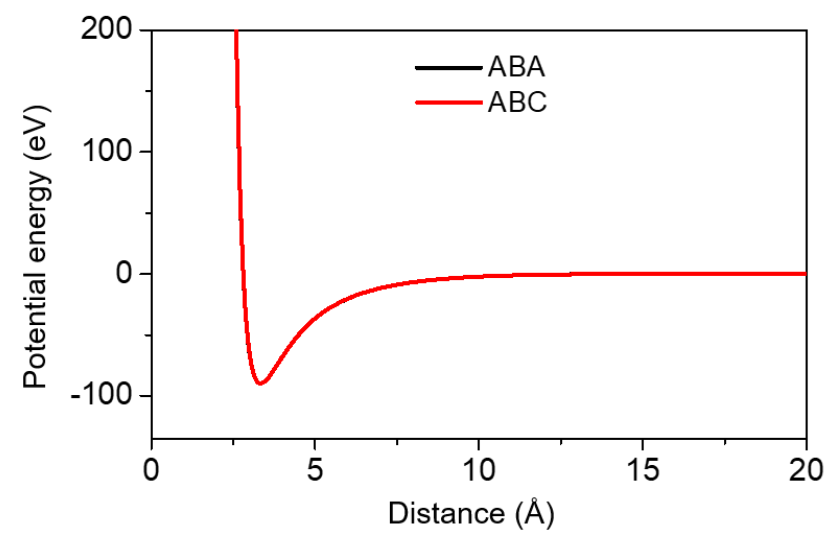

Figure S6. Calculated potential energy between the AFM tip and ABA- or ABCstacked TLG.
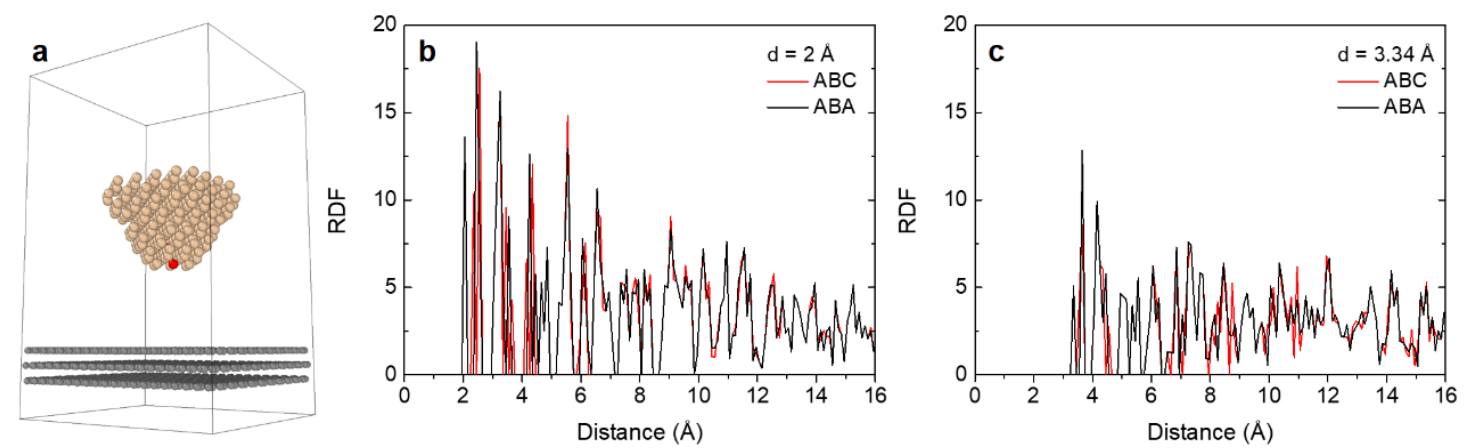

Figure S7. The atomic radial distribution function (RDF) between the (a) tip and graphene with (b) the minimum separation distance of $2 \AA$ and (c) the equilibrium distance of $3.34 \AA$. As can be seen, the neighboring atom distribution around the tip for the $\mathrm{ABA}$ and $\mathrm{ABC}$ stacking are different, hence the interactive potential energy between the tip and graphene are different for different stacking orders. 
Table S2. Comparison between calculation (cal.) and experiment of phase shift in a trilayer graphene with $\mathrm{ABA}$ and $\mathrm{ABC}$ stacking from Figure $1 \mathrm{k}$ in the main text.

\begin{tabular}{|c|c|c|c|c|c|c|c|c|c|c|}
\hline Stacking & $E_{D}(e V)$ & Q & $\mathrm{K}(\mathrm{N} \cdot \mathrm{m})$ & $\mathrm{A}_{0}(\mathrm{~nm})$ & $\mathrm{A}_{\mathrm{t}}(\mathrm{nm})$ & $\sin \varphi(\mathrm{cal})$. & $\varphi($ cal. $)$ & $\Delta \varphi$ (cal.) & $\varphi($ exp.) & $\Delta \varphi(\exp )$. \\
\hline $\mathrm{ABA}$ & 89.7015 & & & & & 0.4076 & 88.38 & & 40.9 & \\
\hline $\mathrm{ABC}$ & 89.6843 & 10.5 & 200 & 1.0 & 0.4 & 0.1712 & 88.13 & 0.25 & 40.7 & 0.20 \\
\hline
\end{tabular}
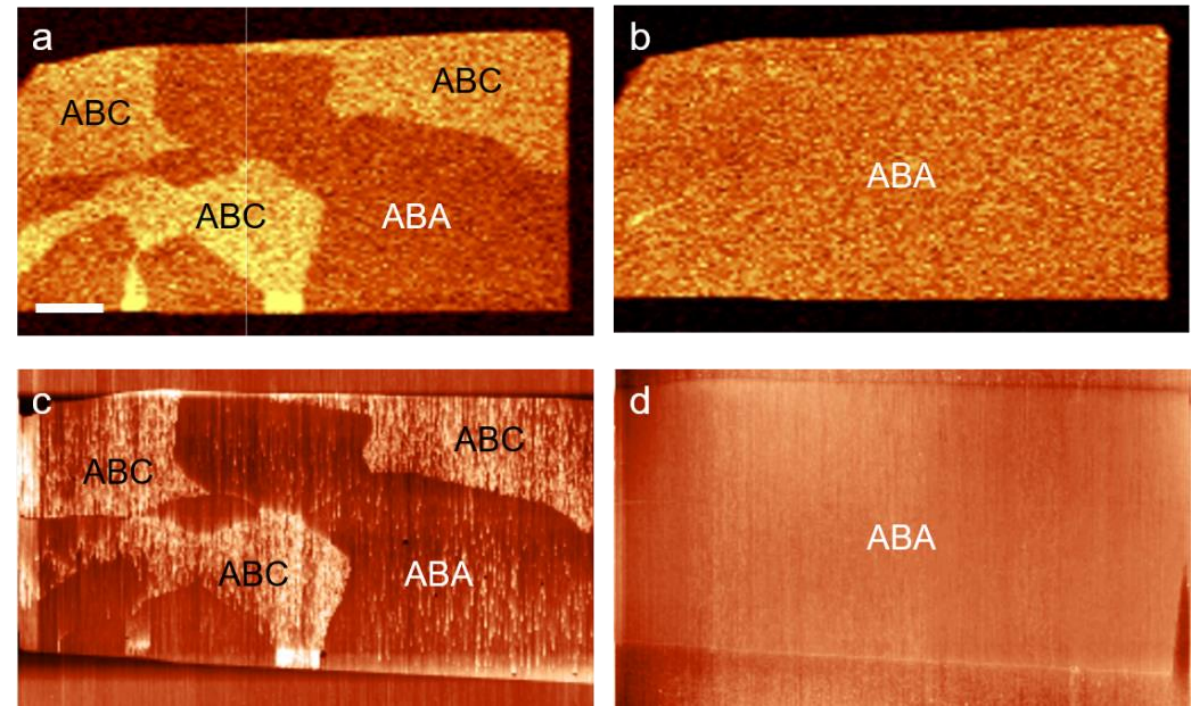

Figure S8. Raman images of FLG before (a) and after (b) the tapping of AFM tip.

AFM phase images of FLG before (c) and after (d) the tapping of AFM tip. The ABCstacked FLG domains can be completely erased and transformed to ABA stacking after the tapping of AFM tip. The scale bar is $10 \mu \mathrm{m}$. 

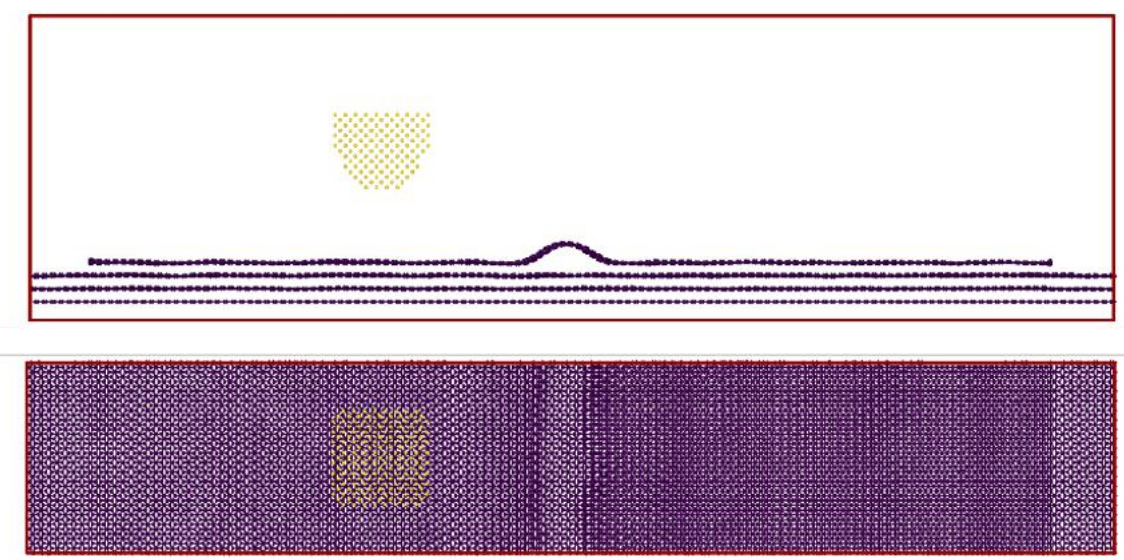

Figure S9. Simulation system for the phase transition. For tip-driven phase transition simulation, the box size is $28.48 \mathrm{~nm} \times 4.98 \mathrm{~nm} \times 8 \mathrm{~nm}$. The tapping speed is constant as $1 \AA /$ fs, and the tapping amplitude is $18 \AA$, with vertical distance ranging from $2 \AA$ to $20 \AA$. The horizontal distance between tip and domain wall is $50 \AA$. The wrinkle at the middle is the domain wall, the left and right side are ABA-stacked and ABCstacked FLG domains, respectively.

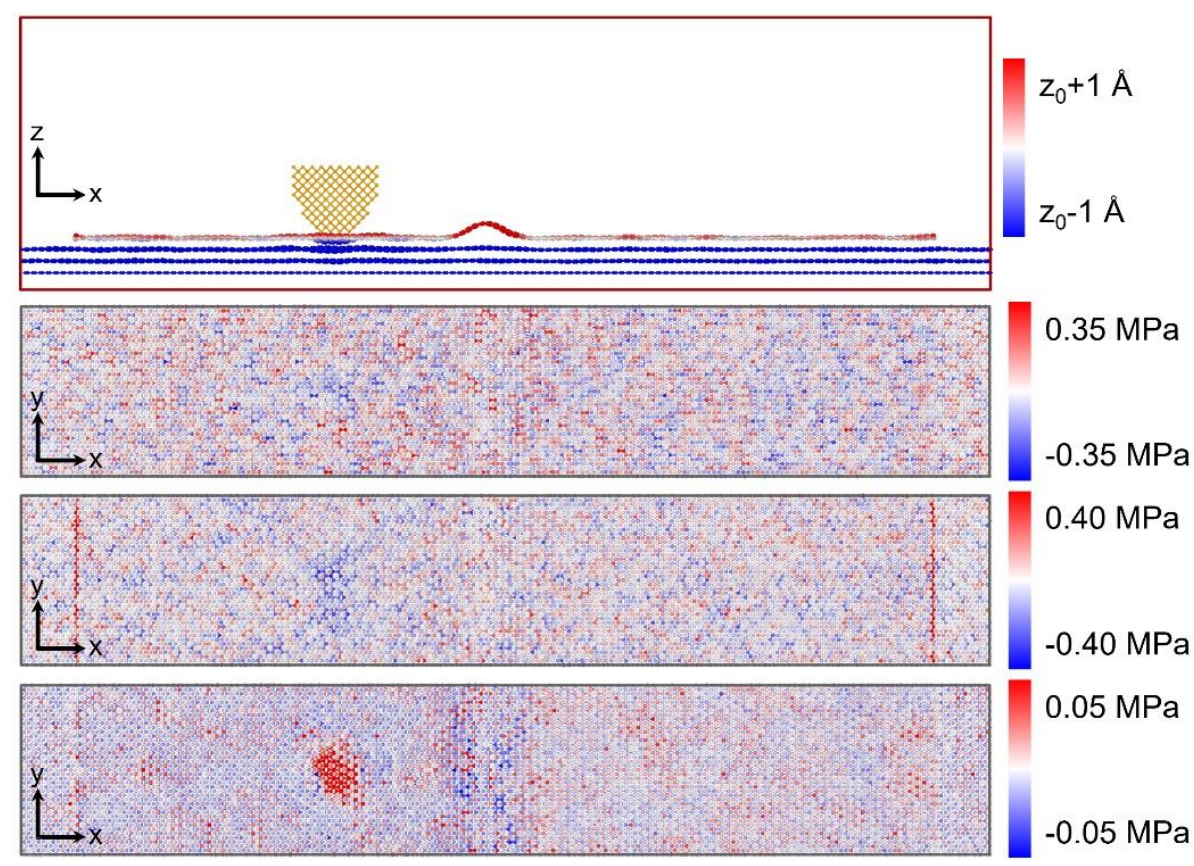

Figure S10. Stress analysis in FLG under the tapping of the AFM tip. (a) A snapshot structure when the tip moves towards the top layer graphene. The graphene sample 
shows obvious out-of-plane strains under the pression of tip. (b d) The stress map of three tensors $\sigma_{\mathrm{xx}}, \sigma_{\mathrm{yy}}$, and $\sigma_{\mathrm{zz}}$. Compare with in-plane stress, out-of-plane stress shows the significant increase of local $\sigma_{\mathrm{zz}}$ at the position where the tip locates due to the repulsive force between tip and graphene.

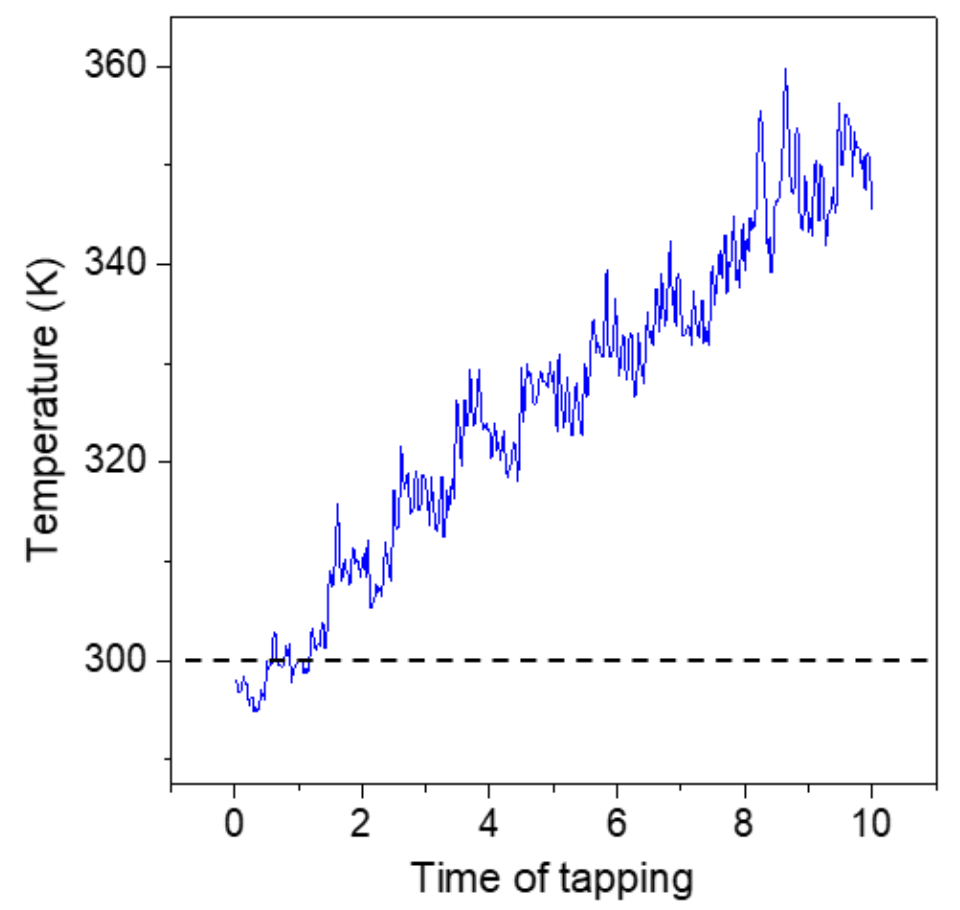

Figure S11. Average temperature of upper layer graphene under the tapping of the AFM tip. Under the one-by-one tapping of AFM tip, the graphene gains energy, hence the average temperature of upper layer shows stepwise increase. The highest value of average temperature during the whole phase transition process is about $350 \mathrm{~K}$. 
Table S3. The comparison of settings of two simulations

\begin{tabular}{ccccc}
\hline $\begin{array}{c}\text { Simulation } \\
\text { system }\end{array}$ & $\begin{array}{c}\text { Tip diameter } \\
(\mathrm{nm})\end{array}$ & $\begin{array}{c}\text { Tapping magnitude } \\
(\mathrm{nm})\end{array}$ & $\begin{array}{c}\text { Sample } \\
\text { width }(\mathrm{nm})\end{array}$ & $\begin{array}{c}\text { In-plane tip-DW } \\
\text { distance }(\mathrm{nm})\end{array}$ \\
\hline A & 2 & 1.8 & 4.98 & 5 \\
\hline B & 2 & 1.8 & & 6 \\
\hline
\end{tabular}
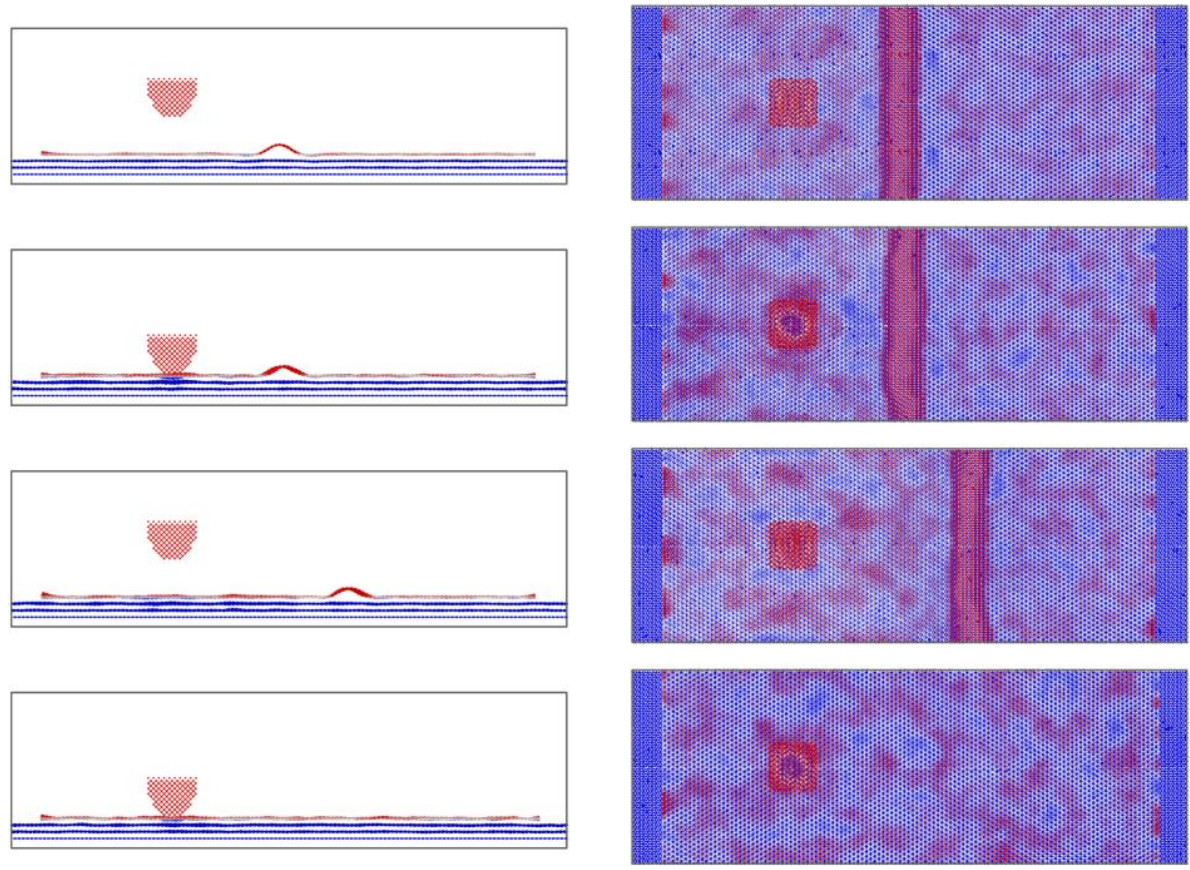

Figure S12. MD simulation snapshots of system with larger sample width and inplane tip-DW distance (simulation system B in Table S3). From top to bottom, the snapshots from top to bottom are the initial state, $8^{\text {th }}$ tapping, $10^{\text {th }}$ tapping, and $15^{\text {th }}$ tapping respectively. As can be seen, the ABC-ABA structural phase transition also happens for system B. The physical pictures of system B are the same as those of system A. However, there are two differences: (1) the time of tapping to trigger the movement of DWs in system B is larger than that in system A; (2) the moving speed of DWs in system B is slower than system A. 


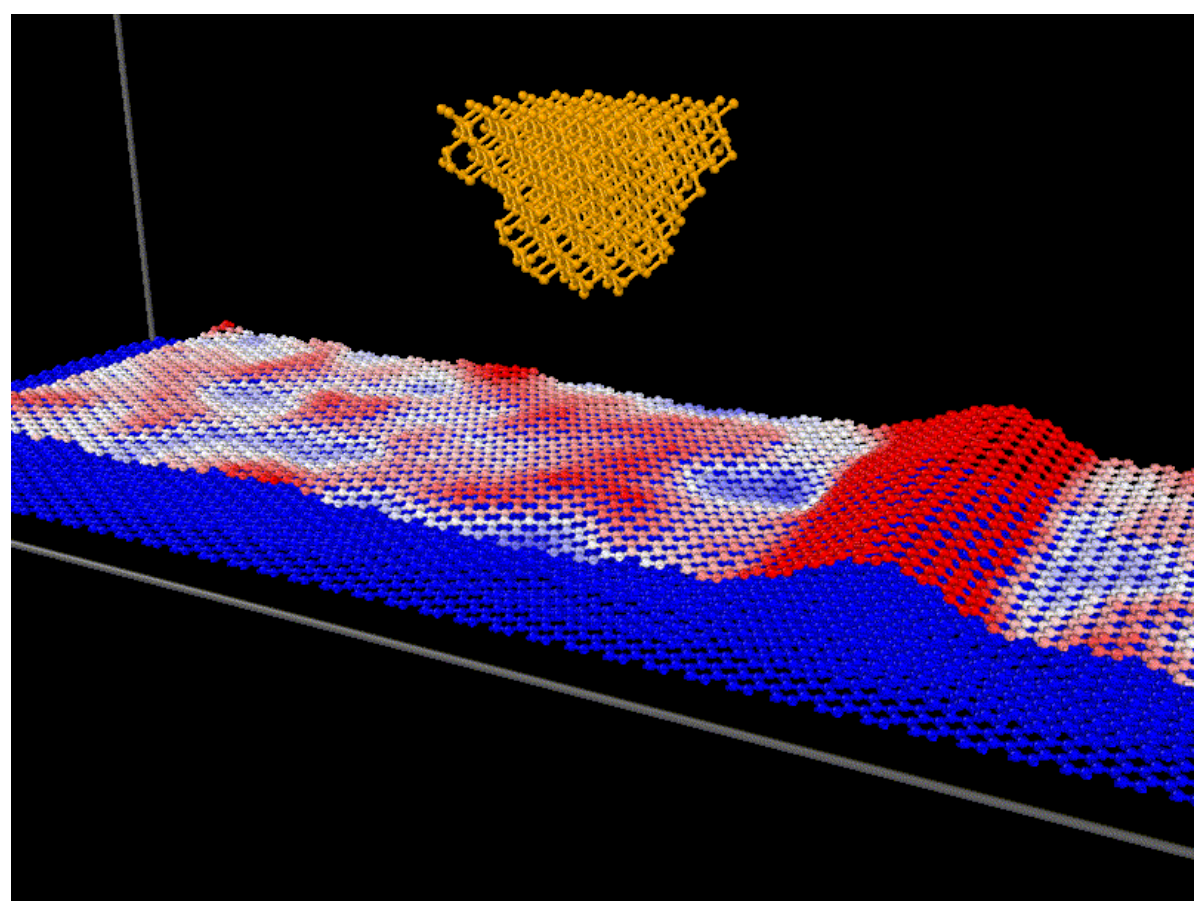

Movie S1. Enlarged perspective view of atomic structure near the tip during one tapping process. The tapping of tip generates ripples which spreads away from the position where the tip locates.

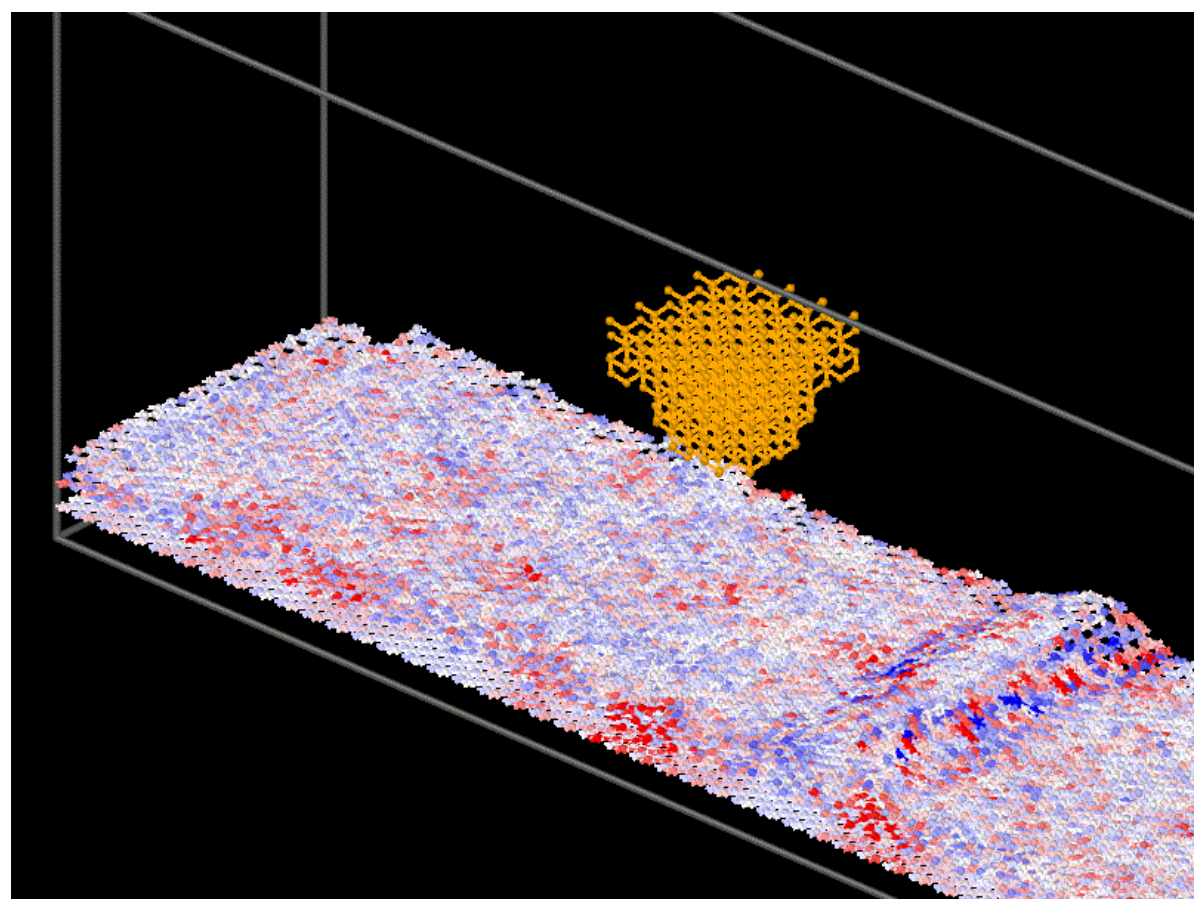


Movie S2. Enlarged perspective view of $\sigma_{\mathrm{zz}}$ stress profile near the tip during one tapping process. The evolution of $\sigma_{\mathrm{zz}}$ stress propagates toward the domain wall, indicating that the phase transition is driven by transverse mechanical waves.
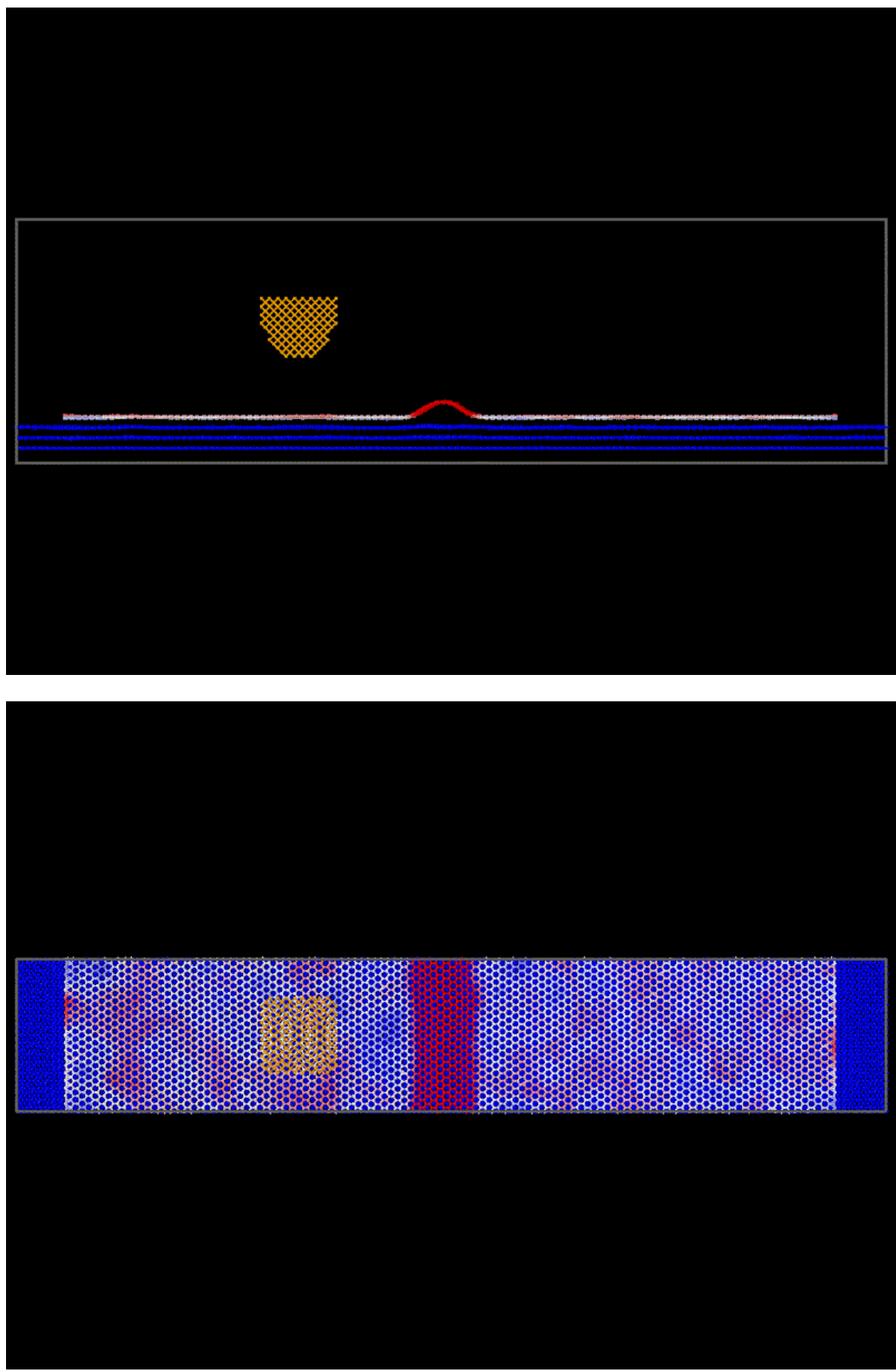
Movie S3 4. Front view and top view of ABC-to-ABA structural phase transition process. Tapping of AFM tip generate transverse mechanical waves, which keep lashing against the domain wall between $\mathrm{ABA}$-stacked and $\mathrm{ABC}$-stacked graphene, then push the domain wall moving toward the edge, finally complete the phase transition.

\section{REFERENCES}

(1) Plimpton, S. Fast parallel algorithms for short-range molecular dynamics. J. Comput. Phys. 1995, 117, 1-19.

(2) Stukowski, A. Visualization and analysis of atomistic simulation data with OVITO-the Open Visualization Tool. Modelling Simul. Mater. Sci. Eng. 2010, 18, 015012.

(3) Tersoff, J. Modeling solid-state chemistry: Interatomic potentials for multicomponent systems. Phys. Rev. B. 1989, 39, 5566-5568.

(4) Lindsay, L. and Broido, D. A. Optimized Tersoff and Brenner empirical potential parameters for lattice dynamics and phonon thermal transport in carbon nanotubes and graphene. Phys. Rev. B. 2010, 81, 205441.

(5) Kolmogorov, A. N. and Crespi, V. H. Registry-dependent interlayer potential for graphitic systems. Phys. Rev. B. 2005, 71, 235415.

(6) Ouyang, W.; Azuri, I.; Mandelli, D.; Tkatchenko, A.; Kronik, L.; Urbakh, M. and Hod, O. Mechanical and Tribological Properties of Layered Materials under High Pressure: Assessing the Importance of Many-Body Dispersion Effects. J. Chem. Theory Comput. 2020, 16, 666-676. (7) Rappé, A. K.; Casewit, C. J.; Colwell, K.; Goddard III, W. A. and Skiff, W. M. UFF, a full periodic table force field for molecular mechanics and molecular dynamics simulations. J. Am. Chem. Soc. 1992, 114, 10024-10035. 